\title{
Thermal diffusivity measurement of copper nanofluid using pulsed laser thermal lens technique
}

\author{
R. Zamiri \\ B. Z. Azmi \\ azmizak@gmail.com
}

\section{Shahril Husin}

\section{G. Zamiri}

\section{H. A. Ahangar}

\section{Z. Rizwan}

\author{
Department of Physics, Faculty of Science, Universiti Putra Malaysia, 43400 UPM Serdang, Selangor, \\ Malaysia \\ Department of Physics, Faculty of Science, Universiti Putra Malaysia, 43400 UPM Serdang, Selangor, \\ Malaysia \\ Advanced Materials and Nanotechnology Laboratory, Institute of Advanced Technology, Universiti \\ Putra Malaysia, 43400 UPM Serdang, Selangor, Malaysia \\ Department of Physics, Faculty of Science, Universiti Putra Malaysia, 43400 UPM Serdang, Selangor, \\ Malaysia \\ Department of Physics, Faculty of Science, Universiti Putra Malaysia, 43400 UPM Serdang, Selangor, \\ Malaysia \\ Faculty of Science, Islamic Azad University, Najafabad Branch, Isfahan, Iran
}

Department of Physics, Faculty of Science, Universiti Putra Malaysia, 43400 UPM Serdang, Selangor, Malaysia

The pulsed laser thermal lens technique was used to study the thermal diffusivity of fluids containing copper nanoparticles (Cu-NPs) prepared by $\gamma$-irradiation method. The samples were prepared for the different concentrations of Cu precursor at 20.KCy dose. A Q-switched Nd-YAG pulsed laser of wavelength 532.nm was used as an excitation source and He-Ne laser was used as a probe beam in the present thermal lens experiment. It was found that the thermal diffusivity of the solution depends on the density of Cu-NPS.

[DOI: http://dx.doi.org/10.2971/jeos.2012.12022]

Keywords: Copper nanoparticles, thermal lens, thermal diffusivity, pulsed laser

\section{I NTRODUCTION}

Metal NPs with various unique applications are very interesting materials for the researchers. Some important applications of metal NPs are in electronic, optical components [1,2], as targeted thermal agents in medical therapies and drug delivery, high functioning heat transfer fluids in automotive electronic cooling systems and in micro-channel heat sinks [3]-[8]. Especially, Cu-NPs have attracted scientists due to its application as catalysis, lubrication $[9,10]$ and conductive films [11]. The synthesis of Cu-NPs is very difficult due to its easy oxidation and aggregation. Few methods like photochemical [12], laser ablation [13, 14], microemulsions [15] are being used to synthesis Cu-NPs. Much literature is available which presents the fabrication of NPs in pure water. However, mostly studies reported the difficulties in the fabrication of Cu-NPs $[13,16]$. But the preparation of NPs by gamma radiation method has significant advantage that there are no impurities like $\mathrm{Cu}$ oxide as compared to the chemical reduction method.

The poor heat transfer properties of conventional fluid such as mineral oil, ethylene glycol and water was the big challenge for heating and cooling system in many industrial processes. On the other hand from long time ago it was known that the thermal conductivity of these fluids can be increased by suspending ultrafine metallic or nonmetallic solid powders in- side them. This idea originated from this fact that most of solid material has higher thermal conductivity in comparison with liquids. In recent decades this idea still has been applying on fluid but this time by using solid in nanometer dimension to form nanofluid and so far interesting thermal transport properties of nanofluid has been addressed by many comprehensive review articles and books.

One of the sensitive and accurate techniques to measure the thermal diffusivity of material is the thermal lens (TL) technique. This technique based on absorption of irradiated Gaussian beam as an excitation source and non-radiative relaxation of the excited molecules which causes formation of the TL inside the sample [17]. On the other hand the other beam which is called probe beam is used to probe the TL signal. The intensity of the probe beam will be detected by a detector which was placed behind a pinhole. The change of intensity is directly related to thermo-optical parameters of sample.

So far the ability of this technique for measurement on the thermal diffusivity of nano-fluids was confirmed by many researchers [18]-[21]. In this work, we used pulsed laser TL technique to measure the thermal diffusivity of fluids containing $\mathrm{Cu}-\mathrm{NPs}$ which were prepared by $\gamma$-irradiation method. 


\section{EXPERIMENT}

All chemicals and reagents were purchased from Sigma-Aldrich. $\mathrm{Cu}$ chloride $\left(\mathrm{CuCl}_{2} \cdot 2 \mathrm{H}_{2} \mathrm{O}, 99 \%\right)$ and polyvinylpyrrolidone (PVP, MW=29000, 95\%) were used as $\mathrm{Cu}$ precursor and stabilizer agent, respectively. Double distilled water was used as a solvent for preparing of all samples. PVP (4.0 g) was dissolved in $100 \mathrm{ml}$ of water and stirred for $30 \mathrm{~min}$ at room temperature to obtain a colorless solution. The prepared PVP solution was divided to 5 parts to make the samples at different $\mathrm{Cu}$ concentration. The different amount of $\mathrm{CuCl}_{2}$ was dissolved in these PVP solutions for the preparation of various samples with different $\mathrm{Cu}$ molar concentration $3.0 \times 10^{-3}(\mathrm{~S} 1), 5.0 \times 10^{-3}$ (S2), $7.0 \times 10^{-3}$ (S3), $1.0 \times 10^{-2}(\mathrm{~S} 4)$ and $5.0 \times 10^{-2}$ (S5) $\mathrm{M}$ under constant stirring conditions. Samples were stirred for 2 hours to obtain the homogeneous solution at room temperature. Each sample was irradiated by $\gamma$-irradiation at a $20 \mathrm{KGy}$ dose.

The prepared $\mathrm{Cu}$-NPs were characterized with double beam UV-vis spectrometer (Shimadzu), and transmission electron microscopy (TEM, Hitachi model H-7100). The size distribution of particles was obtained using UTHSCA software (Ver. 3). We also measured the density of $\mathrm{Cu}$ NPs inside the solution using atomic absorption spectroscopy (S Series).

Schematic diagram of the experimental set up is shown in Figure 1 . The measurements were carried out using a Q-switched Nd:YAG pulsed lasers (SL400/SL800 system) with $532 \mathrm{~nm}$ wavelength, $10 \mathrm{~ns}$ duration as an excitation light source and a He-Ne laser of wavelength $632.8 \mathrm{~nm}$ with the output power less than $1 \mathrm{~mW}$ as a probe beam. The excitation light beam was focused by a lens of $50 \mathrm{~cm}$ focal length onto a liquid sample contained in quartz cuvette positioned vertically at its focal point.

The probe beam was focused by a lens of $25 \mathrm{~cm}$ focal length and cuvette cell located around a distance of $\sqrt{3} z_{c}\left(z_{c}\right.$ is the confocal distance of the probe beam) from the focal point. The transmitted beam was detected by a photodiode detector (PD) which was placed behind the aperture. An optical bandpass filter (F) was placed in front of aperture to remove the excitation beam. The PD output was coupled to the digital storage oscilloscope (LeCroy 9100A $400 \mathrm{MHZ}$ ) to capture the TL signal. The LabVIEW software was used to record the TL data from oscilloscope. The data was normalized with respect to TL signal at time $t=0$. The probe beam intensity at the detector can be written as $[22,23]$ :

$$
\begin{gathered}
\Phi=\frac{I_{t}-I_{\infty}}{I_{t}}=\Phi_{t=0} \frac{1}{\left[1+2 n\left(t / t_{c}\right)\right]^{2}} \\
\Phi_{t=0}=\frac{I_{t=\infty}-I_{t=0}}{I_{t=0}}
\end{gathered}
$$

where, $I_{\infty}$ is the steady state TL signal, $I_{0}$ and $I_{t}$ are TL signal at time $t=0$ and time $=t$, respectively. The value of $n$ can be determine by measuring the dependence of TL signal on laser energy $E$ of the pump laser pulse using the relation,

$$
\frac{I_{t=0}-I_{t=\infty}}{I_{t=0}} \propto E^{n^{\prime}}
$$

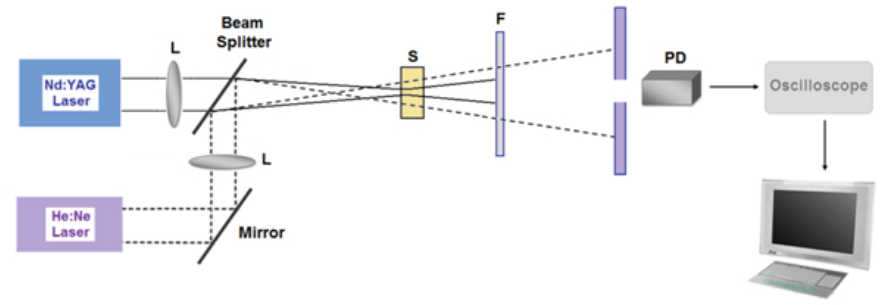

FIG. 1 Schematic diagram of experimental set-up ( $L=$ Lens; $S=$ Sample; $F=$ Filter; $P D=$ Photodiode detector).

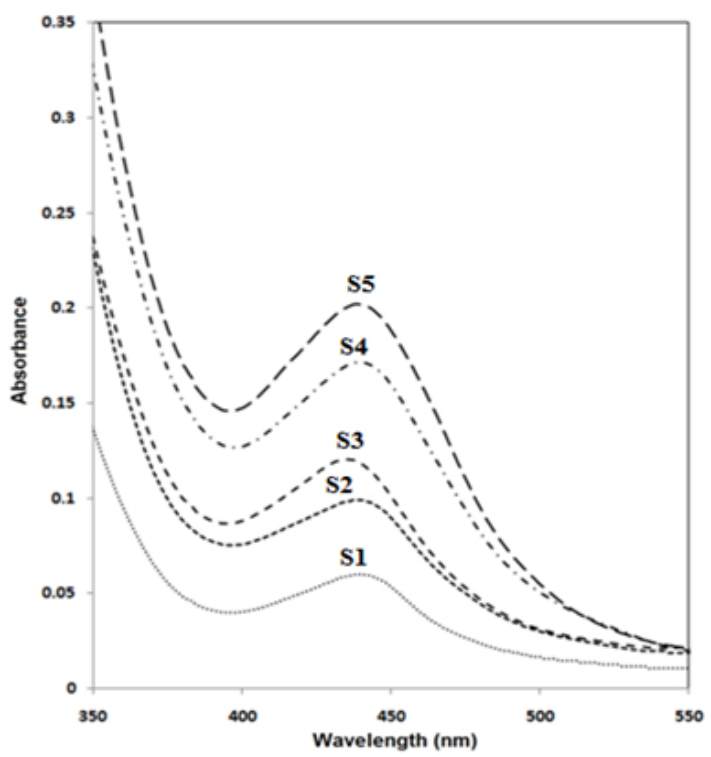

FIG. 2 UV-vis spectra of the synthesized Cu-NPS at different concentration.

The characteristic time constant $t_{c}$ is related to the thermal diffusivity and beam radius $\left(\omega_{e}\right)$ through the equation,

$$
D=\frac{\omega_{e}^{2}}{4 t_{c}}
$$

After finding $t_{c}$ by fitting Eq. (1) to the normalized TL time evolution data, thermal diffusivity can be calculated from the Eq. (4).

\section{RESULTS AND DISCUSSION}

Color of the solution changed from colorless to yellow-green after $\gamma$ - radiation which confirmed the formation of $\mathrm{Cu}-\mathrm{NPs}$. The $\mathrm{Cu}-\mathrm{NPs}$ have a surface plasmon resonance (SPR) peak from 435 to $440 \mathrm{~nm}$ [24] as shown in the UV-vis absorption spectra, (Figure 2). It is clear from the figure that the intensity of the plasmon peaks is related to the density of NPs inside the sample. This result was further confirmed by atomic absorption spectroscopy in which shows that during the process of $\gamma$-irradiation, the converting of $\mathrm{Cu}$ ion to $\mathrm{Cu}$-NPs followed the amount initial $\mathrm{Cu}$ precursor inside the sample.

The particle size of the Cu-NPs was confirmed by TEM image and particle size histogram. It was observed from the TEM images, the produced NPs were well dispersed inside the fluids and non-agglomerated. The average particle size for all the samples was obtained below $5 \mathrm{~nm}$. The average particle size 


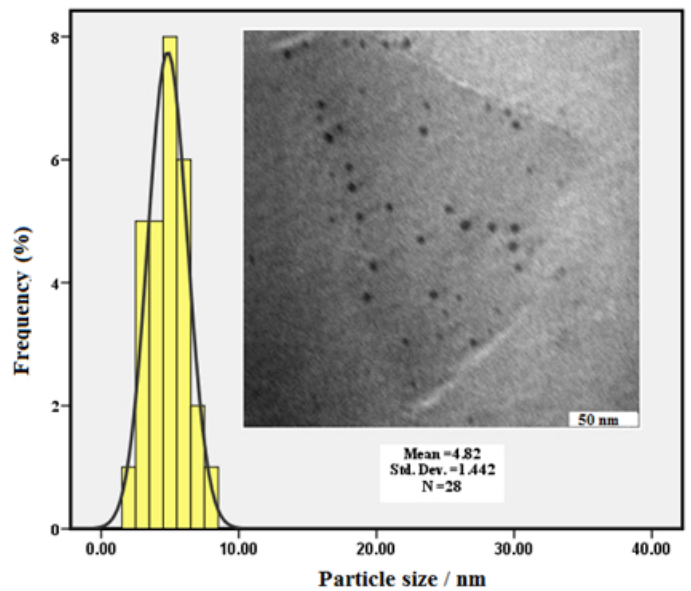

FIG. 3 Typical TEM image particle size distribution of synthesized Cu-NPs (S3).

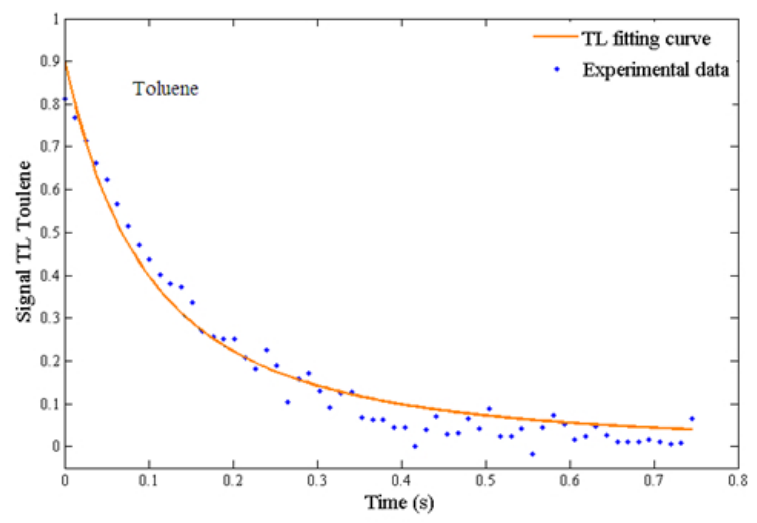

FIG. 4 The normalized TL time evolution signal for toluene. Solid line corresponds to the best fit of Eq. (1) to the TL experimental data. The values of $t_{c}$ obtained from the figure is $0.394 \mathrm{~s}$.

of Cu-NPs for sample S3 was about $4.82 \mathrm{~nm}$ with a narrow size distribution as shown in Figure 3.

The TL signal variation with laser power showed a linear dependence for all the samples. This suggests that one photon absorption happens by mean of $n=1$ as explained by Eq. (1). The calculated value of toluene thermal diffusivity, $D_{\text {Toluene, }}$ from Eq. (4) by inserting the obtained $t_{c}=0.394 \mathrm{~s}$ is $(10.880 \pm 0.003) \times 10^{-4} \mathrm{~cm}^{2} / \mathrm{s}$ and it agrees quite well with the literature value, $10.90 \times 10^{-4} \mathrm{~cm}^{2} / \mathrm{s}$ [25]. The small difference, $0.2 \%$, from literature idicates that the present TL setup is quite accurate in measuring this thermal parameter. The source of error was mostly contributed from the fitting. A reference sample with known thermal diffusivity was used to determine the thermal diffusivity of the unknown sample to eliminate the uncertainty in the determination of beam radius. Toluene was used as reference sample in this experiment (Figure 4). Therefore,

$$
D=D_{\text {Toluene }} \frac{t_{\text {c,Toluene }}}{t_{c}}
$$

We measured the thermal diffusivity of glycerol with toluene as a reference to confirm the accuracy of our experimental set up. Figure 5 shows the normalized TL time evolution signal for glycerol sample where the solid line corresponds

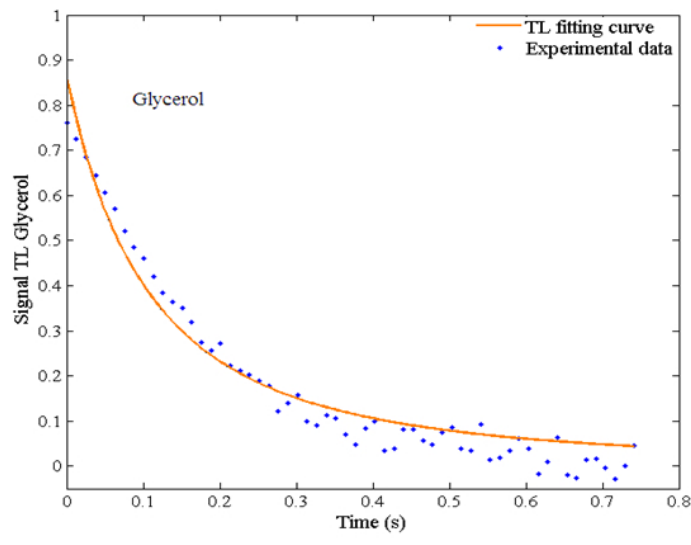

FIG. 5 The normalized TL time evolution signal for glycerol.

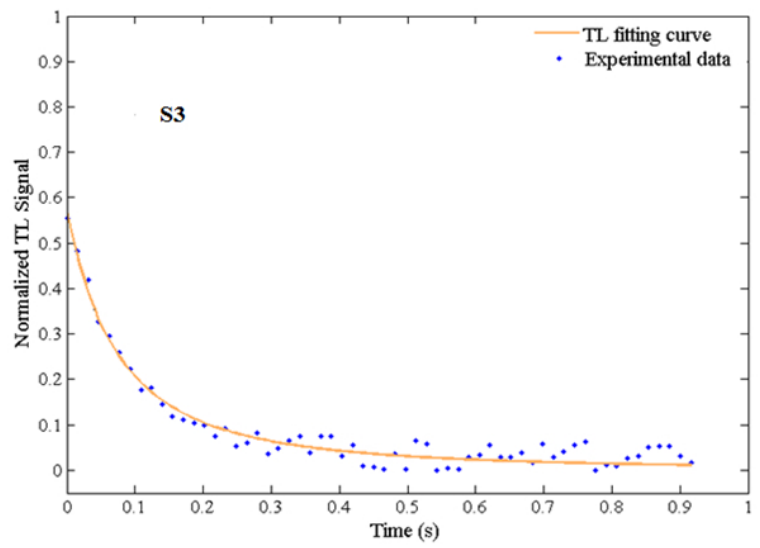

FIG. 6 The normalized TL time evolution signal for $\$ 3$ sample. Solid line corresponds to the best fit of Eq. (1) to the TL experimental data.

to the best fitting to Eq. (1). The values of $\Phi_{0}$ and $t_{c}$ are $(0.861 \pm 0.052)$ and $(0.431 \pm 0.045)$ sec, respectively. The calculated thermal diffusivity $(D)$ value is $(9.491 \pm 0.075) \times 10^{-4} \mathrm{~cm}^{2} / \mathrm{s}$. The difference is around $5 \%$ as compared with the value reported in the literature, $10.00 \times 10^{-4} \mathrm{~cm}^{2} / \mathrm{s}$ [25]. This indicates that our TL experimental set-up is accurate enough and satisfactory for the measurement of the thermal parameters.

Figure 6 shows the TL signal for sample S3. The values of $\Phi_{0}$ and $t_{c}$ are found to be $(0.572 \pm 0.033)$ and $(0.300 \pm 0.033)$ sec obtained from the fitting curve. Thus, the calculated value of thermal diffusivity for sample (S3) is $\left(13.6 \pm 0.9 \times 10^{-4}\right)$ $\mathrm{cm}^{2} / \mathrm{s}$.

Similar TL signals were obtained for other samples and their corresponding values of $\Phi_{0}, t_{c}$ and $D$ are presented in Table 1 .

The behavior of thermal diffusivity in term of particle density has been depicted in Figure 7. As expected, our results show that thermal diffusivity increases with the increase of particle concentration inside the fluid.

The best interpretation for this phenomenon is that, absorption of exciting laser light by nanoparticle generates oscillat- 


\begin{tabular}{|c|c|c|c|c|}
\hline Sample & $\begin{array}{c}\text { Density of } \\
\text { Cu-NPs (mg/L) }\end{array}$ & $\Phi_{t=0}$ & $t_{c}$ (s) & $\mathbf{D}\left(10^{-4} \mathrm{~cm}^{2} / \mathrm{s}\right)$ \\
\hline Glycerol & - & $0.9 \pm 0.05$ & $0.43 \pm 0.04$ & $9.5 \pm 0.9$ \\
\hline S1 & 0.73 & $0.82 \pm 0.09$ & $0.37 \pm 0.03$ & $11.1 \pm 0.9$ \\
\hline S2 & 4.39 & $0.94 \pm 0.04$ & $0.34 \pm 0.02$ & $12.1 \pm 0.9$ \\
\hline S3 & 6.09 & $0.57 \pm 0.03$ & $0.30 \pm 0.03$ & $13.6 \pm 0.9$ \\
\hline S4 & 6.98 & $0.71 \pm 0.06$ & $0.28 \pm 0.02$ & $14.6 \pm 0.9$ \\
\hline S5 & 8.01 & $0.88 \pm 0.04$ & $0.27 \pm 0.02$ & $15.0 \pm 1.0$ \\
\hline
\end{tabular}

TABLE 1 The fitting parameters $\Phi_{0}$ and $t_{c}$ and the calculated thermal diffusivity, $D$, for all the samples.

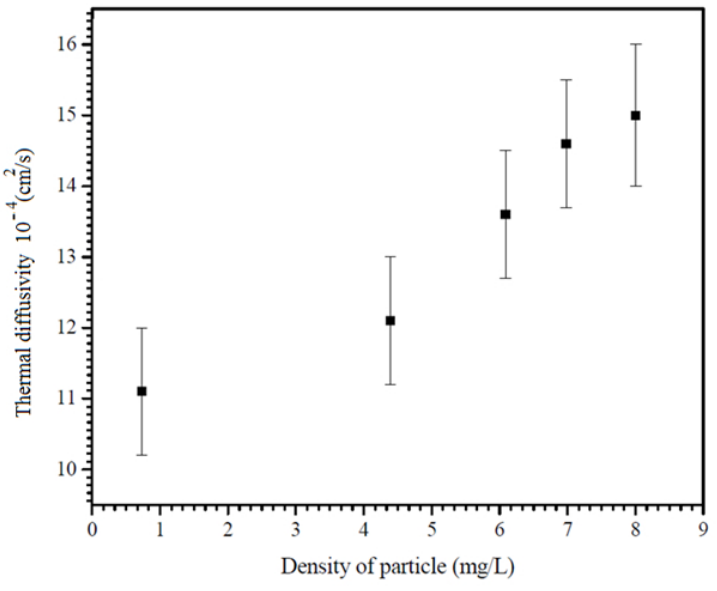

FIG. 7 Thermal diffusivity of the samples as a function of particle density.

ing electron which are called hot electron. These hot electrons rapidly transfer their energy to the particle crystal lattice by electron-phonon scattering. This thermal energy finally transfers to surrounding liquid by phonon scattering at particles boundary. Therefore, when the NPs density is increased in solution, the intensity of optical absorption peaks and in continue thermal diffusivity of fluids were increased.

\section{CONCLUSION}

In summary, we have prepared Cu-NPs in PVP solution by $\gamma$-irradiation method at $20 \mathrm{KGy}$. The thermal diffusivity of fluids containing $\mathrm{Cu}-\mathrm{NPs}$ was measured by pulsed laser thermal lens technique and the obtained results showed that thermal diffusivity of the solutions increases with the increase of $\mathrm{Cu}$ NPs density. The main reason to choose this technique for our measurements is because of its sensitivity to sample thermal properties variation and also avoiding large errors from experimental parameters. On the other hand this technique has an important advantage that is simple linear relation between two measurable parameters, as allow us to directly measure the value of thermal diffusivity.

\section{ACKN OWLEDGEMENTS}

The authors are grateful to the Ministry of Higher Education of Malaysia for supporting this work under Research University Grant No. 05-01-09-0754RU.

\section{References}

[1] D. I. Gittins, D. Bethell, D. J. Schiffrin, and R. J. Nichols, "A nanometre-scale electronic switch consisting of a metal cluster and redox-addressable groups," Nature 408, 67-69 (2000).

[2] S. W. Koch, and A. Knorr, "Optics in the nano-world," Science 293, 2217-2218 (2001).

[3] D. S. Wen, and W. Ding, "Natural convective heat transfer of suspensions of titanium dioxide nanoparticles (Nanofluids)," IEEE T. Nanotechnol. 5, 220-227 (2006).

[4] S. P. Jang, and S. U. Choi, "Cooling performance of a microchannel heat sink with nanofluids," Applied Therm. Eng. 26, 2457-2463 (2006).

[5] K. Hamad-Schifferli, J. J. Schwartz, A. T. Santos, S. G. Zhang, and J. M. Jacobson, "Remote electronic control of DNA hybridization through inductive coupling to an attached metal nanocrystal antenna," Nature $415,152-155$ (2002).

[6] C. Loo, A. Lin, L. Hirsch, M. H. Lee, J. Barton, N. Halas, J. West, and R. Drezek, "Nanoshell-Enabled Photonics-Based Cancer Imaging and Therapy," Technol. Cancer Res. T. 3, 33-40 (2004).

[7] D. P. 0. Neal, L. R. Hirsch, N. J. Halas, J. D. Payne, and J. L. West, "Photo-thermal tumor ablation in mice using near infrared absorbing nanoparticles," Cancer Lett. 209, 171-176 (2004).

[8] G. Huttmann, and R. Birngruber, "On the possibility of highprecision photo thermal micro effects and the measurement of fast thermal denaturation of proteins," IEEE J. Sel. Top. Quant. 5, 954962 (1999).

[9] G. Larsen, and S. Noriega, "Dendrimer-mediated formation of CuCu0x nanoparticles on silica and their physical and catalytic characterization," Appl. Catal. A-Gen. 278, 73-81 (2004).

[10] S. Tarasov, A. Kolubaev, S. Belyaev, M. Lerner, and F. Tepper, "Study of friction reduction by nanocopper additive to motor oil," Wear 252, 63-69 (2002).

[11] H. Wang, Y. Huang, Z. Tan, and X. Hu, "Transient mixing characteristic of reactor pressure vessel under pressurized thermal shock," Anal. Chim. Acta 526, 13-17 (2004).

[12] S. Kapoor, and T. Mukherjee, "Photochemical formation of copper nanoparticles in poly n-vinyl pyrrolidone," Chem. Phys. Lett. 370, 83-87 (2003).

[13] P. V. Kazakevich, A. V. Simakin, V. V. Voronov, and G. A. Shafeev, "Laser induced synthesis of nanoparticles in liquids," Appl. Surf. Sci. 252, 4373-4380 (2006).

[14] R. M. Tilaki, A. Irajizad, and S. M. Mahdavi, "Size, composition and optical properties of copper nanoparticles prepared by laser ablation in liquids," Appl. Phys. A 88, 415-419 (2007).

[15] I. Capek, "Preparation of metal nanoparticles in water-in-oil (W/0) 
microemulsions Advances in Colloid and Interface," Science 110, 49-74 (2004).

[16] M. Saito, K. Yasukawa, T. Umeda, and Y. Aoi, "Copper nanoparticles fabricated by laser ablation in polysiloxane," Opt. Mat. 30, 1201-1204 (2008).

[17] J. Shen, R. D. Lowe, and R. D. Snook, “A model for CW laser induced mode-mismatched dual-beam thermal lens spectrometry," Chem. Phys. 165, 385-396 (1992).

[18] R. Zamiri, B. Z. Azmi, E. Shahriari, M. S. Husin, and M. Mahdi, "Thermal diffusivity measurement of silver nanofluid by using thermal lens technique," J. Laser Appl. 23, 042002-042006 (2011).

[19] Q. Xue, and W. M. Xu, "A model of thermal conductivity of nanofluids with interfacial shells," Mater. Chem. Phys. 90, 298-301 (2005).

[20] C. V. Bindhu, S. S. Harilal, V. P. N. Nampoori, and C. P. G. Vallabhan, "Solvent effect on absolute fluorescence quantum yield of rhodamine 6C determined using transient thermal lens technique," Mod. Phys. Lett. B 13, 563-576 (1999).
[21] J. L. J. Perez, J. F. S. Ramirez, R. G. Fuentes, A. Cruz-Orea, and J. L. $H$. Perez, "Enhanced of the R6C Thermal Diffusivity on Aggregated Small Gold Particles," Braz. J. Phys. 36, 1025-1028 (2006).

[22] A. J. Twarowski, and D. S. Kliger, "Multiphoton absorption spectra using thermal blooming theory," Chem. Phys. 20, 253-258 (1977).

[23] A. J. Twarowski, and D. S. Kliger, "Multiphoton absorption spectra using thermal blooming: II. Two-photon spectrum of benzene," Chem. Phys. 20, 259-264 (1977).

[24] L. P. Ding, Y. Fang, "The study of resonance Raman scattering spectrum on the surface of $\mathrm{Cu}$ nanoparticles with ultraviolet excitation and density functional theory," Spectrochim. Acta A 67, 767-771 (2007).

[25] R. C. Weast, CRC Handbook of Chemistry and Physics (CRC Press, Boca Raton, 1987). 